Premature Chromosome Condensation. Application in Basic, Clinical, and Mutation Research

Edited by $\mathbf{P} \mathbf{N}$ Rao, R T Johnson, and K Sperling. (Pp xvi +381 ; figures + tables. $£ 29.80, \$ 45 \cdot 00$.) New York: Academic Press. 1982.

The technique of virus (or PEG) induced cell fusion has led to major advances in several areas of biology, most spectacularly in the hybridoma technique for the production of monoclonal antibodies, and in the use of interspecific cell hybrids for gene mapping. A fascinating but less completely fulfilled and less well-known application of cell fusion is in the study of the cell cycle, and the process of chromosome condensation and decondensation which occurs as cells move from interphase through mitosis into the next interphase.

In 1970, Johnson and Rao made the fundamental observation that mitotic cells have the ability to induce chromosome condensation in other nuclei, which are not at the appropriate stage of the cell cycle, and they named this 'premature chromosome condensation' (PCC). By fusing synchronised cell populations at the appropriate stages of the cycle, chromosomes can be visualised in $G 1, G 2$, and $S$ phase nuclei, alongside the normal mitotic chromosomes. Chromosomes at these various stages have characteristic morphology, for example, $\mathbf{S}$ phase cells produce a specific fragmented appearance. Cytologists and histologists have long recognised this appearance, without understanding it, under the name "chromosome pulverisation'. Pulverised chromosomes are now shown to be a feature of infection by viruses able to mediate cell fusion, producing occasional heterokaryons between mitotic and S phase cells. The G1 and $\mathrm{G} 2$ chromosomes can be banded, although the quality obtained is generally inferior to that of more conventional chromosome preparations.

The technique has been used to study in detail the process of coiling which converts a nucleosome chain into a recognisable chromosome, and this subject is well reviewed. Some progress has been made in characterising a high molecular weight protein or proteins which are heat labile, $\mathrm{Ca}^{+}$-sensitive, and $\mathrm{Mg}^{+}$-dependent, and which appear to play a critical role in various cellular phenomena preceding mitosis, including nuclear membrane breakdown and chromosome contraction. It is fascinating, although perhaps not surprising, that these fundamental cellular processes are common across a wide range of living organisms, and cross-activity has $\frac{\bar{D}}{\overline{0}}$ even been shown between plant and animal cells.

By fusing cells immediately after irradiation or $\stackrel{\mathbb{Q}}{\varrho}$ mutagen exposure, primary effects of chromosome $\approx$ breakage can be separated from the effects of $\vec{\circ}$ subsequent (mis)repair, and without the bias imposed by seeing only those cells capable of $\vec{\omega}$ proceeding into mitosis.

PCC can be induced in meiotic cells, including spermatids, and may open another avenue for $\dot{i}$ exploring the origin of aneuploid gametes, although $\overrightarrow{-}$ the present quality of preparations obtainable $\vec{\nabla}$ would have to be improved. Similarly, the technique could open the way to identifying chromosome abnormalities in other 'difficult' cells, such as the early stages of solid tumours. PCC induced in $\vec{T}$ leukaemic bone marrow cells has produced the $\mathbb{D}$ very important observation that non-cycling normal cells come to rest in early $\mathrm{G} 1$, while malignant cells rest in late G1. This difference can be reliably $\overrightarrow{\vec{\theta}}$ measured, precedes standard haematological signs $\infty$ of active leukaemia, and can provide informatiog of real diagnostic and prognostic value in some leukaemias.

The entire field of PCC seems to be poised on the brink of providing important insights into funda- ֻँ mental aspects of cellular function. Although $\stackrel{\mathbb{\perp}}{\complement}$ hardly a book to be browsed through casually, this $\overrightarrow{\overrightarrow{0}}$ is an important 'state-of-the-art' summary, which $\frac{0}{3}$ should stimulate more people to try their hand $\bar{\partial}$ with this challenging, difficult, but fascinating technique.

\section{Minor Malformations in the Neonate}

By $\mathrm{K}$ Méhes. (Pp 129; figures + tables. $\$ 6 \cdot 00$.) Budapest: Akadémiai Kiadó. 1983.

This interesting little book is partly a research study and partly a small textbook on minor malformations. $N$ The author has examined 4589 liveborn neonates $N$ and noted the incidence of minor malformations. 0 After a brief introduction there is a review of minor $\mathrm{W}$ malformations found in various studies of neonates. After this there is a breakdown of the incidence of minor malformations where infants have had other $\mathbb{Q}$ abnormalities, such as major malformations or $\stackrel{+}{?}$ 'brain damage'.

There then follows a review of 40 or so minor $\frac{\mathrm{O}}{\mathbb{D}}$ 
malformations. For each malformation the incidence is given, followed by simple aids to diagnosis or measurement. A list of syndromes in which the minor malformation is commonly found is given and a note on the overall clinical significance is also provided. This section is the most useful part of the book and provides much information of interest. The lists of syndromes where each specific minor malformation is commonly found are not exhaustive. However, they do cover the more common malformation syndromes. An alphabetical index would have been useful.

In summary, this is a useful book to have on one's shelf to look up the incidence of various minor malformations and to check on methods of measuring or recording these defects. It will not be very useful as an aid to diagnosis of dysmorphic syndromes, but does provide some insight into the clinical significance of minor malformations.

ROBIN M WINTER

\section{Mobile Genetic Elements}

Edited by James A Shapiro. (Pp xvi + 688; figures + tables. $\$ 65$, £43.) London, New York: Academic Press. 1983.

This volume outlines the exciting developments in what is known about the natural history and molecular basis of mobile genetic elements in prokaryotic and eukaryotic organisms. A detailed and comprehensive review of the present state of knowledge and reference list is provided for the various known mobile genetic elements starting, as it should, with the controlling elements in maize, the bacteriophages lambda and $\mathrm{Mu}$, the non-viral mobile elements in bacteria (the insertion sequence (IS) elements and the transposons, Tn3 and Tn10, which confer drug resistance to bacteria), the transposable elements in yeast, the dispersed repetitive DNAs (copia-like, fold-back, and P elements), and hybrid dysgenesis in Drosophila and Agrobacterium tumour induction (crown gall tumour). Four final chapters on retroviruses, flagellar phase variation on Salmonella, yeast mating types, and antigenic variation in Trypanosomes are included because they involve DNA sequences which display behaviour and possess characteristics typical of mobile elements, although they are not always considered in discussions on this topic.

A stated intent of the editor of the text is to enable the non-specialist to begin to understand the biology and genetics of mobile genetic elements. This objective is not achieved in certain cases because although each chapter starts with an introductory section outlining the biology of the particular genetic element being considered, it occasionally requires a greater degree of background knowledge than the non-specialist might be expected to possess in order to be able to understand the experimental details of the system being discussed. Although most chapters include some discussion of similarities in the biology and molecular basis with other mobile genetic elements, the text is not one which can easily be read from cover to cover in the expectation of providing an overview on the whole subject. Each chapter does provide, however, an excellent starting point for a detailed enquiry into what is known about any particular mobile genetic element.

The descriptions of the behaviour, biology, and molecular basis of these mobile genetic elements provokes questions about our understanding of chromosomal structure, genome stability/instability, and gene regulation. Although one is warned several times in the text that our understanding of these elements in eukaryotic systems is still in a relatively primitive state, one cannot help but speculate about their possible role in the reprogramming, restructuring, and relocation of genes and possible aetiology in some syndromes and single gene and chromosomal disorders. Overall, this is a text for the partly initiated rather than the uninitiated.

R F Mueller 